\title{
Prequalification of VDSL2 Customers for G.fast Services
}

\author{
Driton Statovci, Martin Wolkerstorfer, and Sanda Drakulić \\ Institute of Telecommunications, TU Wien, Gußhausstraße 25/E389, 1040 Vienna, Austria \\ Emails:\{driton.statovci, martin.wolkerstorfer, sanda.drakulic\}@nt.tuwien.ac.at \\ Phone: +43-1-58801389-\{214, 213, 69\}; Fax: +43-1-58801389-99
}

\begin{abstract}
In the near future, digital subscriber line (DSL) network operators will start deploying G.fast technology to stay competitive in the broadband market. G.fast provides data rates of up to $1 \mathrm{Gbps}$, which is a 10x higher data rate than offered by the widely deployed very high speed DSL 2 (VDSL2) technology. In this paper we propose a novel algorithm to prequalify existing VDSL2 customers for G.fast services. Motivated by testbed and field trial results we also propose a new loop attenuation parameter for prequalification purposes, namely the geometric loop attenuation (GeoLATN) instead of the "classical" loop attenuation (LATN). G.fast testbed experiments show that on average the difference between the attainable net data rates (AttNDR) prequalfied by our algorithm and reported by G.fast systems is $\mathbf{5 \%}$. Furthermore, on average the GeoLATN-based prequalification outperforms the LATN-based prequalification by $5 \%$ in terms of AttNDR.
\end{abstract}

\section{INTRODUCTION}

The International Telecommunication Union (ITU) has approved a new recommendation for digital subscriber line (DSL) systems called G.fast [1]. It provides aggregate data rates of up to $1 \mathrm{Gbps}$ over a single twisted-pair line, which is a 10x higher data rate than offered by the widely deployed very high speed DSL 2 (VDSL2) technology. G.fast systems uses frequencies up to $106 \mathrm{MHz}$ and a $212 \mathrm{MHz}$ profile is under study. Differently, current VDSL2 systems only exploit $17.7 \mathrm{MHz}$. G.fast systems are designed and tailored for fiber-to-the-distribution-point (FTTDp) and fiber-to-thebuilding (FTTB) deployments. DSL network operators are developing strategies to deploy G.fast technology aiming at increasing the service rates offered to customers and to stay competitive with cable and wireless network operators.

Various loop attenuation values are defined in DSL recommendations, which characterize the quality of twisted-pair lines for DSL services, such as: a) the loop attenuation at a given reference frequency, e.g., $100 \mathrm{kHz}$ or $150 \mathrm{kHz}$ [2]; b) the loop attenuation (LATN), which is calculated as the arithmetic mean of the attenuation over all frequencies/subcarriers of a particular transmission direction [3]; and c) the loop attenuation per band (LATN-pb), which is calculated as the arithmetic mean of the attenuation over all frequencies/subcarriers of a particular frequency band [4]. In the current G.fast recommendation [1], the loop attenuation is still not defined. Since G.fast is using time-division duplexing (TDD), there is no need to specify the loop attenuation per band. We propose a new loop attenuation parameter that we call geometric loop attenuation
(GeoLATN), which is calculated as the geometric mean of the attenuation over all frequencies/subcarriers of a particular transmission direction. Our testbed and field trial evaluation results show that the GeoLATN is better suited to characterize the loop quality in terms of attainable net data rates (AttNDR) than the previously used "classical" LATN.

Single-ended line testing (SELT) can be used to identify the channel and also to prequalify a loop for DSL services [5]. SELT is still not specified for G.fast and it also requires VDSL2 service interruption to be able to identify the channel beyond $17.7 \mathrm{MHz}$ utilized by G.fast technology. In this paper, we propose a novel algorithm to prequalify existing VDSL2 customers for G.fast services. It is an offline lookup table prequalification approach, which is based on AttNDR reported by G.fast systems and GeoLATN. The GeoLATN of a loop to be prequalified is estimated using the reported VDSL2 channel data. The main contributions of this paper are: a) the definition of the GeoLATN parameter to characterize loop quality for G.fast prequalification; b) the development of a novel algorithm to prequalify the existing VDSL2 customers for G.fast services; and c) the performance evaluation of the proposed prequalification algorithm in a testbed with actual VDSL2 and G.fast systems.

The rest of the paper is organized as follows: In Section II we introduce the network model and define the GeoLATN parameter. Section III introduces our algorithm to prequalify VDSL2 customers for G.fast services. Next, in Section IV the testbed experiment results for the proposed algorithm are presented, before summarizing our conclusions in Section V.

\section{Network Model And Definition of Geometric LOOP ATTENUATION}

DSL network operators are deploying VDSL2 systems from central office $(\mathrm{CO})$ or street cabinet $(\mathrm{Cab})$. When a G.fast distribution point unit (DPU) is deployed in an area, we assume that all existing VDSL2 customers are migrated from $\mathrm{CO}$ or $\mathrm{Cab}$ to FTTDp or FTTB as illustrated in Fig. 1. This deployment strategy avoids truck rolls when existing VDSL2 customers are upgraded to G.fast services, because only a modem replacement at the customer side is required and the customer himself can easily install it. Without loss of generality, we assume that G.fast and (vectored or nonvectored) VDSL2 systems are spectrally separated, as shown 


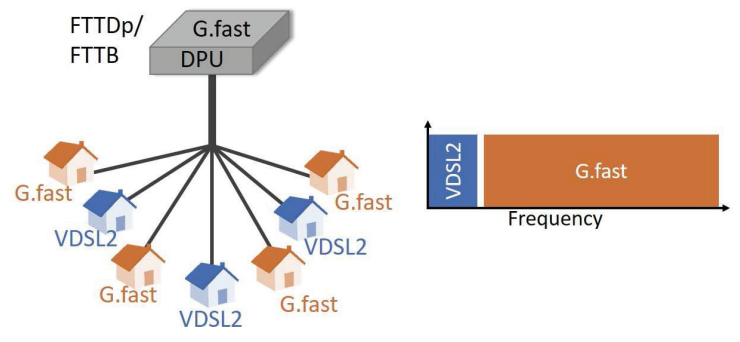

Fig. 1. DSL network with all existing VDSL2 customers migrated to a FTTDp/FTTB node and division of frequency spectrum.

in Fig. 1, to ensure the coexistence of both systems when deployed jointly in the same cable bundle.

As specified in [3, p. 101], the loop attenuation (LATN) is the difference in $\mathrm{dB}$ between the power received at the nearend and that transmitted at the far-end over all subcarriers. Hence, it equals the average of the channel characteristics function $H(f)$ over all subcarriers, where $f$ is the frequency of a subcarrier in $[\mathrm{Hz}]$. LATN, in $\mathrm{dB}$, is defined as

$$
\operatorname{LATN}_{\mathrm{dB}}=-10 \log _{10}\left(\frac{\sum_{c \in C}|H(c \cdot \Delta f)|^{2}}{K}\right),
$$

where $\Delta f, C$, and $K$ denote the subcarrier spacing, the set of subcarrier indexes of a particular transmission direction, and the number of subcarrier indexes in set $C$, respectively. $H(f)$ is reported in linear scale as $\operatorname{Hlin}(f)$ in diagnostics mode or in logarithmic scale as $H \log (f)=10 \log _{10}\left(|H(f)|^{2}\right)$ in initialization mode. The Hlog is defined in Amendments 1 and 2 of the G.fast recommendation [1].

We define the GeoLATN in $\mathrm{dB}$ as the geometric mean of $H(f)$, given by

$$
\operatorname{GeoLATN}_{\mathrm{dB}}=-10 \log _{10}\left(\prod_{c \in C}|H(c \cdot \Delta f)|^{2}\right)^{1 / \mathrm{K}},
$$

where all parameters are the same as those in (1). The GeoLATN can also be calculated as the arithmetic mean of $H \log$ values as

$$
\operatorname{GeoLATN}_{\mathrm{dB}}=-\frac{\sum_{c \in C} H \log (c \cdot \Delta f)}{K} .
$$

Current G.fast systems do not report LATN and GeoLATN parameters, but there are ongoing activities at the ITU to include them into the G.fast recommendation.

\section{VDSL2 Prequalification for G.Fast Services}

\section{A. Lookup Tables for G.fast Attainable Net Data Rate}

We develop a novel algorithm to prequalify existing VDSL2 systems for G.fast services based on the reported VDSL2 channel data. It is based on downstream and upstream lookup tables consisting of AttNDR values reported by G.fast systems and corresponding calculated GeoLATN values. We use GeoLATN as a parameter for loop prequalification because of its better correlation with AttNDR compared to LATN as it is shown in Fig. 2 and 3 for testbed and field trial results,

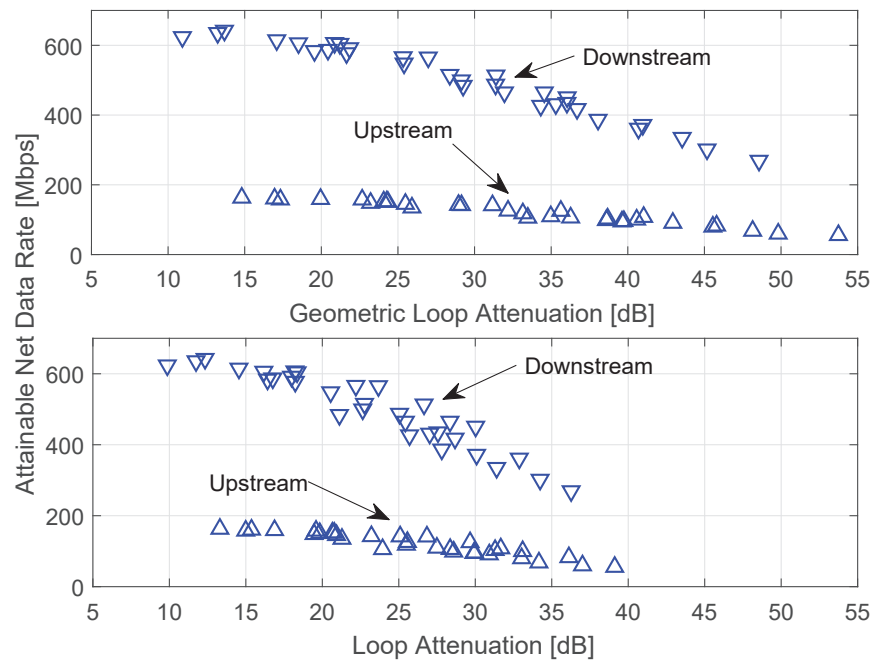

Fig. 2. Testbed G.fast downstream and upstream AttNDR versus GeoLATN/LATN for profile 106a: 21-106 MHz.

respectively. More precisely, since the data in both plots in Fig. 2 can be approximated using a monotonic function, we may choose Spearman's rank correlation [6] to quantify the correlation between the AttNDR and GeoLATN/LATN. For GeoLATN we obtain a correlation coefficient $\rho_{\text {GeoLATN }}=$ $-0.98\left(\mathrm{p}\right.$-value $\left.\mathrm{GeoLATN}_{\text {in }}=3.4 \cdot 10^{-23}\right)$, whereas for LATN we obtain: $\rho_{\text {LATN }}=-0.95\left(\right.$ p-value $\left.{ }_{\text {LATN }}=1.5 \cdot 10^{-17}\right)$. Moreover, to quantify the variability of AttNDR, we search in both plots with a GeoLATN and LATN sliding window width of $2 \mathrm{~dB}$ for the highest range of AttNDR values. For the downstream GeoLATN (Fig. 2, upper plot), the highest range is within the $31-33 \mathrm{~dB}$ window, where the difference between the maximum and minimum AttNDR is less than 49 Mbps (11\%). In the case of LATN (Fig. 2, lower plot) the highest range is within the $26-28 \mathrm{~dB}$ window with a difference of $127 \mathrm{Mbps}(33 \%)$. Similar results are observed in the upstream. In summary, this analysis shows that in both case the correlation is "very strong", but the correlation between the AttNDR and GeoLATN is better.

We also analyzed the field trial results carried out at several locations of a DSL network operator. In total there are 23 active lines in these eight locations. G.fast DPUs are installed at building basements and each DPU has a different number of active lines. Fig. 3 shows the AttNDR reported by G.fast systems versus GeoLATN/LATN parameters. Similar to the testbed results, these field trial results show that the correlation between AttNDR and GeoLATN is better as confirmed in the following by correlation analysis. Spearman's rank correlation for GeoLATN show: $\rho_{\text {GeoLATN }}=-0.91$ and p-value GeoLATN $=3.3 \cdot 10^{-6}$, whereas for LATN: $\rho_{\text {GeoLATN }}=$

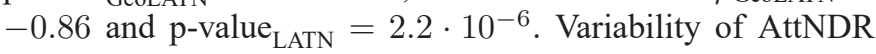
for the GeoLATN (window: 14-15 dB) shows the difference between the maximum and minimum AttNDR is $37 \mathrm{Mbps}$ (7\%), whereas for the LATN (window: $30-31 \mathrm{~dB}$ ) it is 128 Mbps (39\%). These results are similar to the testbed results.

Let us analyze the results marked by red circles in the upper 


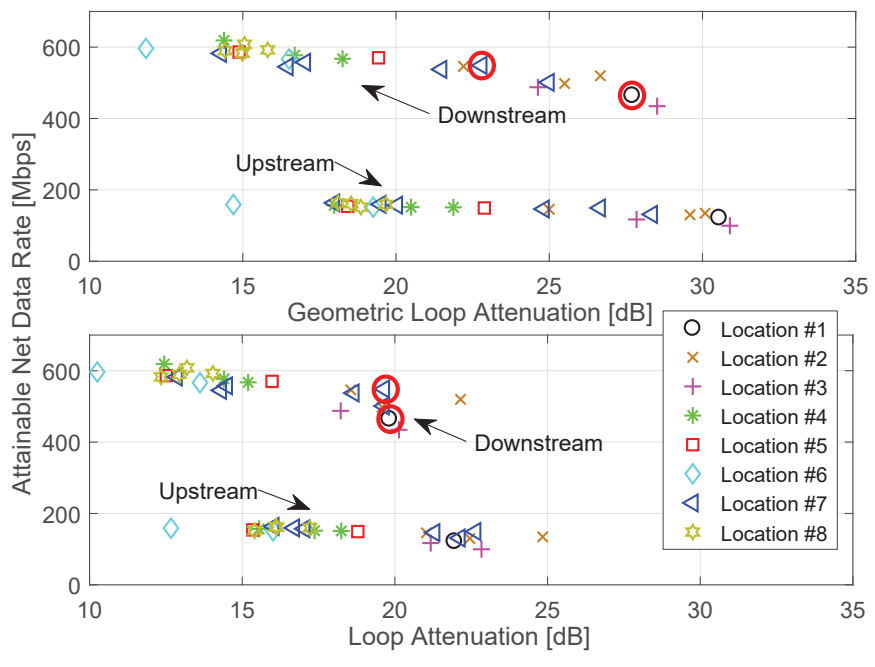

Fig. 3. Field trial G.fast downstream and upstream AttNDR versus GeoLATN/LATN for profile 106a: 21-106 MHz.

and lower plots in Fig. 3, which in fact correspond to the same lines. The Hlog values for these two lines and their respective AttNDR, LATN, and GeoLATN are shown in Fig. 4. The LATN values are nearly the same for these lines although the AttNDR values are quite different, whereas the GeoLATN values do in fact reflect the difference in AttNDR. In the case of LATN, due to the arithmetical mean operation in linear scale (see (1)), larger Hlog values influence the mean value more than the smaller ones. As a result, always when Hlog of different lines cross each other as in Fig. 4 (in practice this is often the case due to the use of different cables), the LATN values are not correctly qualifying the lines in terms of supported AttNDR.

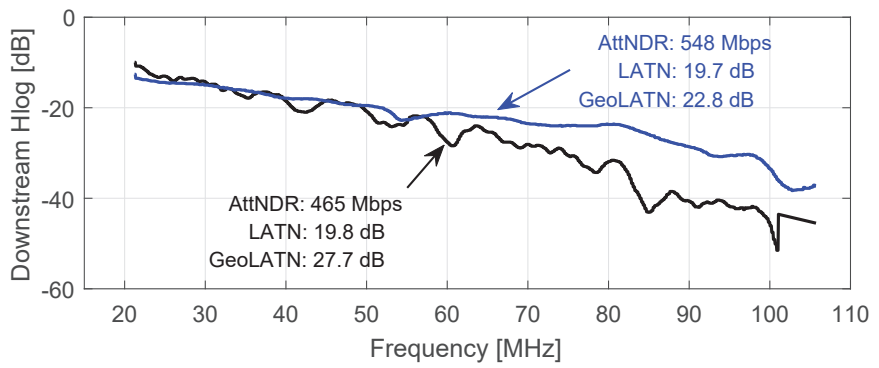

Fig. 4. Downstream Hlog values for the loops marked with red circles in Fig. 3 and the respective AttNDR, LATN, and GeoLATN.

\section{B. Prequalification Algorithm}

We estimate the G.fast channel, $H \log _{\mathrm{G}}^{\text {est }}(f)$ in G.fast frequencies ${ }^{1}$, by using the reported VDSL2 channel data, $H \log _{\mathrm{V}}(f)$. For this purpose we could use any of the twistedpair cable models suitable for G.fast e.g., as specified in [7]. However, the best prequalification results, based on the empirical testbed and field trial data, were obtained by using a

\footnotetext{
${ }^{1}$ In our case the G.fast systems are utilizing frequencies $21-106 \mathrm{MHz}$, but the algorithm can also be used when the start frequency is at $2.2 \mathrm{MHz}$ or when the profile $212 \mathrm{MHz}$ is used.
}

generalization of the simple twisted-pair square root model given by

$$
H \log _{\mathrm{G}}^{\mathrm{est}}(f)=a f^{b},
$$

where the parameters $a$ and $b$ are calculated by curve fitting of (4) using VDSL2 Hlog data. Having estimated the channel as in (4), the GeoLATN is calculated as in (3), where instead of $H \log (f)$ we use $H \log _{\mathrm{G}}^{\text {est }}(f)$. Examples of $H \log$ data reported by actual VDSL2 systems and the corresponding fitted model in (4) are shown in Fig. 5. We see that the reported Hlog values in lower and higher frequencies are not following our used model. To have a good estimate, independently for each transmission direction, we should only use frequencies/subcarriers that minimize the Hlog estimation error in G.fast frequencies. Analysis on our available data has shown that for downstream the estimation error is minimized if we only use $H \log$ in frequencies between $3 \mathrm{MHz}$ and $16 \mathrm{MHz}$.

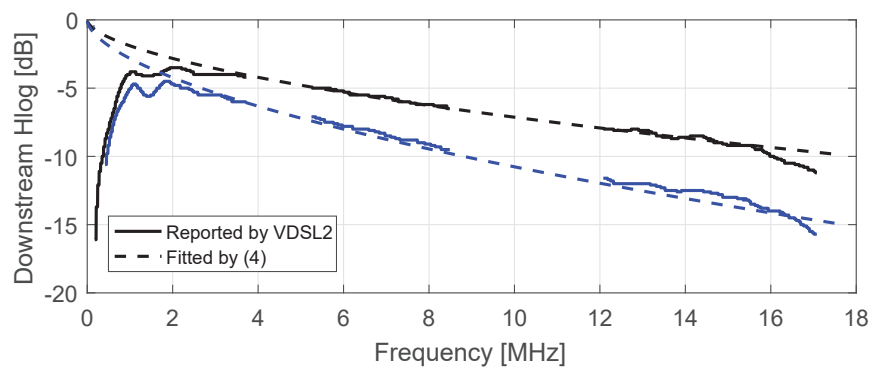

Fig. 5. VDSL2 reported Hlog values and fitted models by using a generalization of the simple twisted-pair square root models as specified in (4).

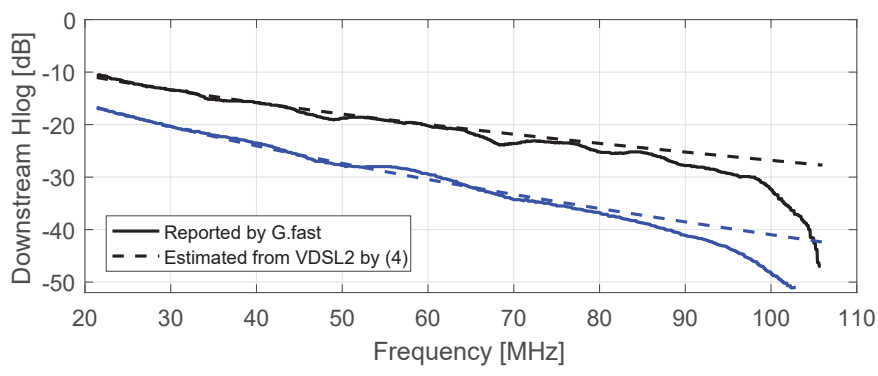

Fig. 6. G.fast reported Hlog values and the estimated values based on extrapolation of the VDSL2 data shown in Fig. 5.

For illustration purpose, Fig. 6 shows the data reported by the actual G.fast systems on the same lines and the model in (4) fitted based on VDSL2 Hlog data shown in Fig. 5. These results indicate that the estimation is sufficiently accurate and there is a good match between the reported and estimated values. For this case, there is a mismatch between the reported and estimated values in high frequencies (above $80 \mathrm{MHz}$ ). This is because the Hlog values reported by the G.fast systems include also the transfer functions of the analogue front-end at the transmitter and receiver sides.

Algorithm 1 summarizes the developed approach to prequalify VDSL2 customers for G.fast services. DSL network operators should start with downstream and upstream G.fast 


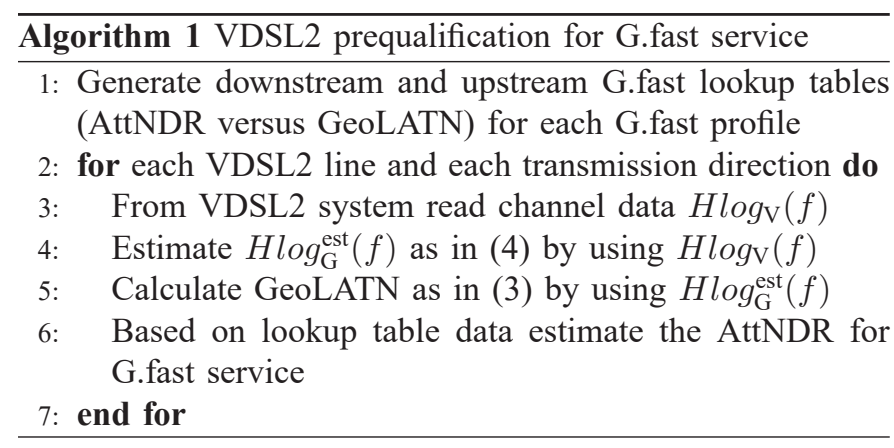

lookup tables (AttNDR versus GoeLATN) generated from testbed data and later update them using field data. Lookup tables are generated for a given GeoLATN step e.g., $0.1 \mathrm{~dB}$ such that e.g., $95 \%$ of all reported G.fast AttNDR are above the values in the lookup table.

\section{Testbed Results}

We have performed different tests at a DSL network operator's G.fast testbed in order to analyze the performance of Algorithm 1. All VDSL2 lines are configured with band plan '998ADE17-M2X-A', which is typically used for street cabinet deployments. All G.fast lines are configured with 106a profile, power spectral density notching up to $21 \mathrm{MHz}$ to protect VDSL2 systems, and a downstream/upstream TDD ratio of 4:1. The G.fast testbed consists of a 20-pair $0.5 \mathrm{~mm}$ quad installation cable $(10 \mathrm{~m}$ or $100 \mathrm{~m}$ long); shielded and unshielded two-pair $0.5 \mathrm{~mm}$ drop cables of $30-115 \mathrm{~m}$ lengths laid out in a tree topology; a G.fast DPU, which supports also vectoring VDSL2; and 8 G.fast and 8 VDSL2 modems as illustrated in Fig. 7. Tests are carried out such that at a time either VDSL2 or G.fast modems are connected at shielded or unshielded cables for $10 \mathrm{~m}$ or $100 \mathrm{~m}$ installation cable.

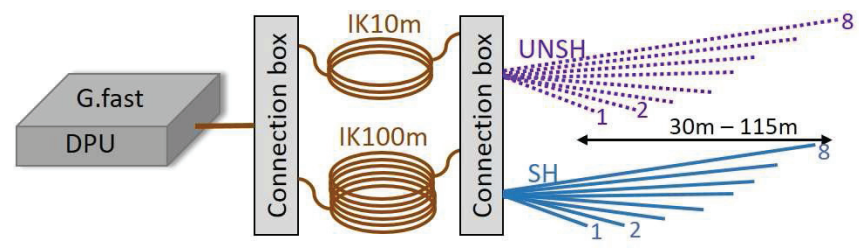

Fig. 7. Illustration of G.fast testbed. IK denotes the installation cables. SH and UNSH denote shielded and unshielded drop cables, respectively.

We calculate LATN and GeoLATN parameters as in (1) and (3) based on reported G.fast Hlog data on subcarriers between 21-106 MHz. Unfortunately, on some lines the G.fast systems did not reported $H \log$ values over all used subcarriers, e.g., $H \log$ values in higher frequencies were missing. Since we are working with (arithmetic and geometric) mean values of persubcarrier attenuation values, for the sake of fair comparison among the lines, we should always compare vectors with equal lengths. For this purpose we have estimated the missing $H \log$ values by means of interpolation/extrapolation with the first order twisted-pair attenuation model as specified in [7].
Additionally, the G.fast reported downstream and upstream $H \log$ data are not equal and the difference can be up to a couple of $\mathrm{dB}$ as illustrated in Fig. 8 for a specific line. Therefore, we use the downstream GeoLATN parameter to also generate the upstream G.fast lookup tables and only downstream VDSL2 Hlog for prequalification. Note that in general, the VDSL2 and G.fast Hlog upstream data should be used for upstream prequalification in Algorithm 1.

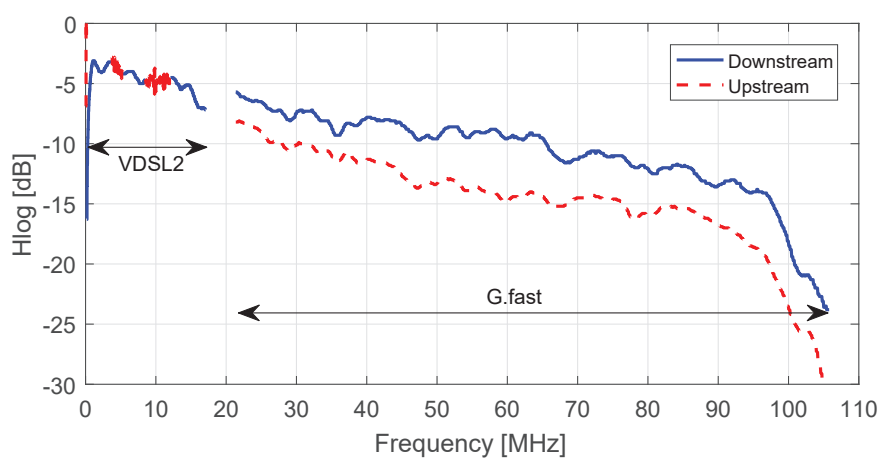

Fig. 8. Illustration of difference between G.fast and VDSL2 reported downstream and upstream $H \log$ values.

Fig. 9 and 10 show the G.fast downstream and upstream AttNDRs for different testbed configurations versus the downstream GeoLATN and LATN, respectively. We use these data to generate the downstream and upstream lookup tables, which are also shown in the respective plots. In these particular cases, we generate the lookup tables such that the reported G.fast AttNDR values are on or above the lookup curves.

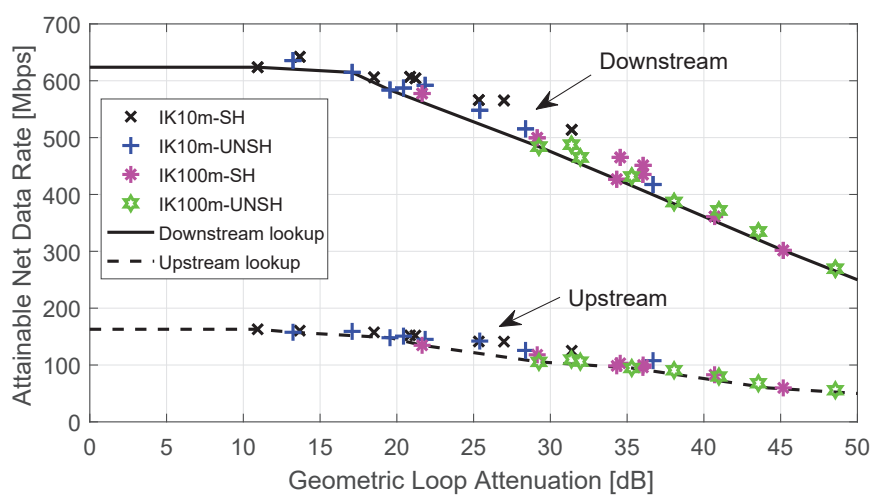

Fig. 9. G.fast downstream and upstream AttNDR versus downstream GeoLATN for different cable configurations including downstream and upstream lookup curves.

To evaluate the performance of Algorithm 1 we connect VDSL2 modems to the lines and configure the G.fast DPU with the specified VDSL2 band plan. Based on VDSL2 Hlog data we prequalify the lines for G.fast services and the results are summarized in Fig. 11 for both downstream and upstream transmission directions. The results indicate that there is a good match between the reported and prequalified AttNDR with a mean absolute percentage error (MAPE) of $4 \%$ and $6 \%$ in downstream and upstream, respectively. On average 


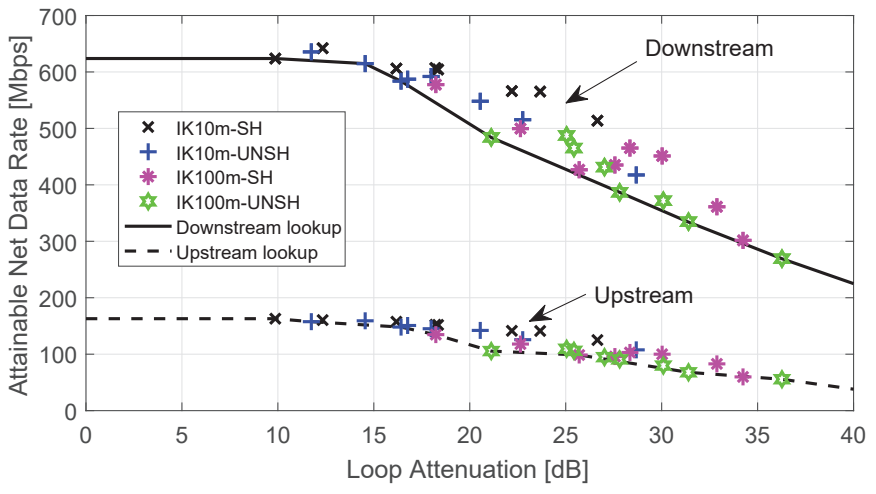

Fig. 10. G.fast downstream and upstream AttNDR versus downstream LATN for different cable configurations including downstream and upstream lookup curves.

the downstream prequalification is better than the upstream one, because we are using downstream data for upstream prequalification. Furthermore, the downstream prequalification is better for short lines $(\mathrm{IK}=10 \mathrm{~m})$, whereas for upstream no trend can be recognized.

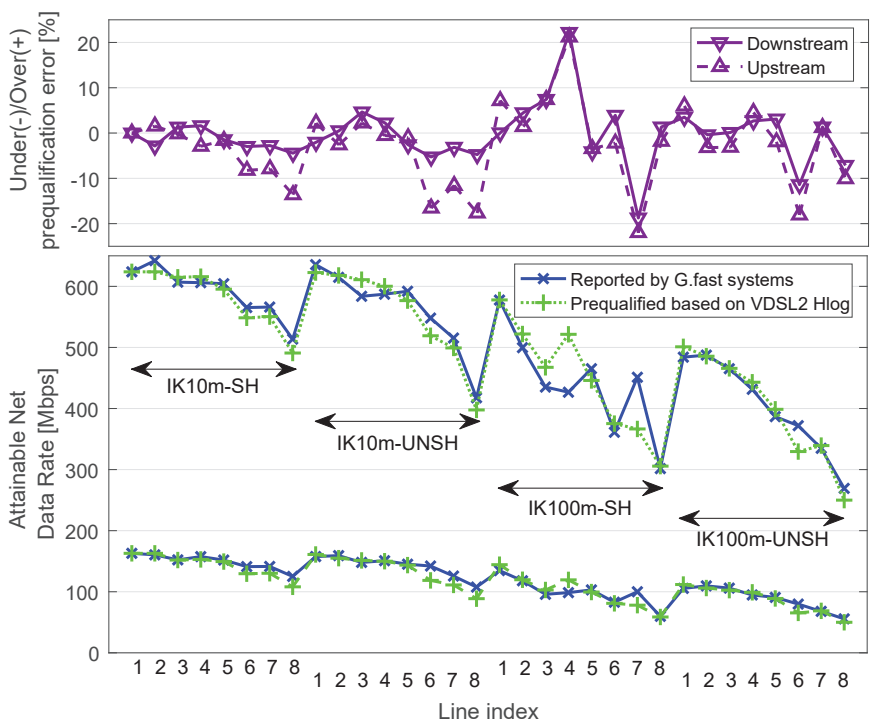

Fig. 11. Performance of VDSL2 prequalification for G.fast services for different testbed cable configurations based on the GeoLATN parameter.

Fig. 12 compares the performance results between GeoLATN- and LATN-based prequalification. We also use Algorithm 1 for LATN-based prequalification, but with LATN parameter and the lookup tables shown in Fig. 10. These prequalification results show that LATN-based AttNDR values are always below GeoLATN-based values. Therefore, when GeoLATN-based prequalification is equal or smaller than $0 \%$ (perfect or under prequalification), which is true in most of the cases then it is outperforming LATN-based prequalification. Otherwise, when GeoLATN-based values are above $0 \%$ (over prequalification, which arises when Hlog is overestimated) then LATN-based prequalification might outperform GeoLATN-based one e.g., line 4 for IK100m-SH. On average our results show that the GeoLATN-based prequalification in terms of AttNDR outperforms LATN-based prequalification by $5 \%$ (MAPEs difference) in both transmission directions.

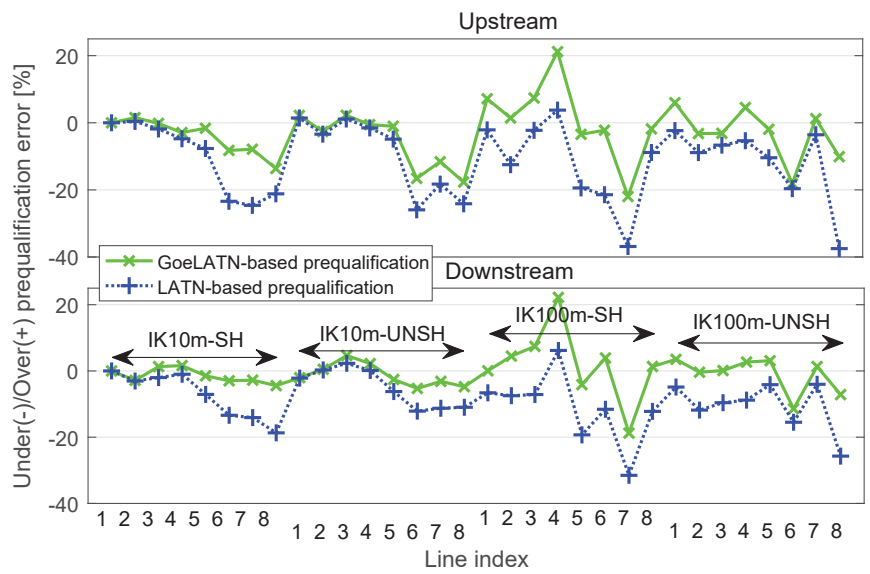

Fig. 12. Performance comparison between GoeLATN-based and LATN-based prequalifications for different testbed cable configuration.

\section{COnClusions}

We developed and evaluated the performance of a novel algorithm to prequalify existing VDSL2 customers for G.fast services. It is based on VDSL2 Hlog data and G.fast attainable net data rate (AttNDR) lookup tables. Furthermore, we propose a new loop attenuation parameter for prequalification purposes, namely the geometric loop attenuation (GeoLATN), instead of the "classical" loop attenuation (LATN). Testbed and field trial results show that there is a better correlation between AttNDR and GeoLATN than between AttNDR and LATN. G.fast testbed experiments show that on average the difference between the prequalified AttNDR and reported by G.fast systems is $4 \%$ and $6 \%$ in downstream and upstream, respectively. Furthermore, on average the GeoLATN-based prequalification in terms of AttNDR outperforms LATN-based prequalification by $5 \%$ in both transmission directions.

\section{ACKNOWLEDGMENTS}

This work has been funded by A1 Telekom Austria AG.

\section{REFERENCES}

[1] ITU-T, "Fast access to subscriber terminals (G.fast) -- Physical layer specification," G.9701, Dec. 2014.

[2] J. J. Werner, "The HDSL environment," IEEE Journal on Selected Areas in Communications, vol. 9, no. 6, pp. 785-800, Aug 1991.

[3] ITU-T, "Asymmetric digital subscriber line transceivers 2 (ADSL2)," G.992.3, Jan. 2005.

[4] - "Very high speed digital subscriber line transceivers 2 (VDSL2)," G.993.2, Jan. 2015.

[5] S. Galli and D. L. Waring, "Loop makeup identification via single ended testing: Beyond mere loop qualification," IEEE Journal on Selected Areas in Communications, vol. 20, no. 5, pp. 923-935, Jun 2002.

[6] C. Spearman, "The proof and measurement of association between two things," American Journal of Psychology, vol. 15, no. 1, pp. 72-101, Jan 1904.

[7] D. Acatauassu, S. Höst, C. Lu, M. Berg, A. Klautau, and P. O. Börjesson, "Simple and causal copper cable model suitable for G.fast frequencies," IEEE Transactions on Communications, vol. 62, no. 11, pp. 4040-4051, Nov 2014. 